Old Dominion University

ODU Digital Commons

2016

\title{
Seebeck Coefficient Enhancement of ALD PbTe/PbSe Nanolaminate Structures Deposited Inside Porous Silicon Templates
}

\author{
Xin Chen \\ Old Dominion University, xchen011@odu.edu \\ Pengtao Lin \\ Old Dominion University, pxlin002@odu.edu \\ Kai Zhang \\ Old Dominion University, kzhang@odu.edu \\ Helmut Baumgart \\ Old Dominion University, hbaumgar@odu.edu \\ Brian Geist
}

See next page for additional authors

Follow this and additional works at: https://digitalcommons.odu.edu/ece_fac_pubs

Part of the Physics Commons

\section{Original Publication Citation}

Chen, X., Lin, P. T., Zhang, K., Baumgart, H., Geist, B., \& Kochergin, V. (2016). Seebeck coefficient enhancement of ALD PbTe/PbSe nanolaminate structures deposited inside porous silicon templates. ECS Journal of Solid State Science and Technology, 5(9), P503-P508. doi:10.1149/2.0151609jss

This Article is brought to you for free and open access by the Electrical \& Computer Engineering at ODU Digital Commons. It has been accepted for inclusion in Electrical \& Computer Engineering Faculty Publications by an authorized administrator of ODU Digital Commons. For more information, please contact digitalcommons@odu.edu. 
Authors

Xin Chen, Pengtao Lin, Kai Zhang, Helmut Baumgart, Brian Geist, and Vladimir Kochergin 


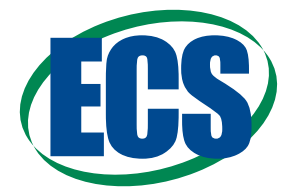

\title{
Seebeck Coefficient Enhancement of ALD PbTe/PbSe Nanolaminate Structures Deposited inside Porous Silicon Templates
}

\author{
Xin Chen, ${ }^{\mathrm{a}, \mathrm{b}, *}$ Pengtao Lin, ${ }^{\mathrm{a}, \mathrm{b}, *}$ Kai Zhang, ${ }^{\mathrm{a}, \mathrm{b}, * *}$ Helmut Baumgart, ${ }^{\mathrm{a}, \mathrm{b}, * *, \mathrm{z}}$ Brian Geist, ${ }^{\mathrm{c}}$ \\ and Vladimir Kochergin ${ }^{\mathrm{c}}$
}

${ }^{a}$ Department of Electrical and Computer Engineering, Old Dominion University, Norfolk, Virginia 23529, USA

${ }^{b}$ Applied Research Center at Thomas Jefferson National Accelerator Laboratories, Newport News, Virginia 23606, USA

${ }^{c}$ MicroXact Inc., Blacksburg, Virginia 24060, USA

\begin{abstract}
In this paper, lead chalcogenide based thermoelectric nanolaminate structures were fabricated by alternately depositing $\mathrm{PbTe}$ and $\mathrm{PbSe}$ ALD layers on regular planar silicon wafers and on microporous silicon templates. Lead bis(2,2,6,6-tetramethyl-3,5-heptanedionato) $\left(\mathrm{Pb}\left(\mathrm{C}_{11} \mathrm{H}_{19} \mathrm{O}_{2}\right)_{2}\right)$, plus (trimethylsilyl) telluride $\left(\left(\mathrm{Me}_{3} \mathrm{Si}\right)_{2} \mathrm{Te}\right)$ and (trimethylsilyl) selenide $\left(\left(\mathrm{Me}_{3} \mathrm{Si}\right)_{2} \mathrm{Se}\right)$ were used as the chemical ALD precursors for lead, tellurium and selenium, respectively. The Seebeck coefficient in horizontal direction (parallel direction to the surface) to the multiple layered $\mathrm{PbTe} / \mathrm{PbSe}$ nanolaminate structures was measured by an MMR Seebeck system, and benchmarked against the Seebeck coefficient in the vertical direction to the sample surface. The results of the Seebeck measurements clearly indicate that the multiple layered $\mathrm{PbTe} / \mathrm{PbSe}$ nanolaminate structures synthesized by ALD on microporous silicon templates exhibit significantly increased Seebeck coefficients in both horizontal and vertical directions, in stark contrast to the case when the same ALD thermoelectric nanolaminates are grown on regular planar bulk Si substrates.

(C) The Author(s) 2016. Published by ECS. This is an open access article distributed under the terms of the Creative Commons Attribution 4.0 License (CC BY, http://creativecommons.org/licenses/by/4.0/), which permits unrestricted reuse of the work in any medium, provided the original work is properly cited. [DOI: 10.1149/2.0151609jss] All rights reserved.
\end{abstract}

Manuscript submitted May 12, 2016; revised manuscript received July 8, 2016. Published July 29, 2016. This was Paper 1057 presented at the Phoenix, Arizona, Meeting of the Society, October 11-15, 2015.

As a green renewable technology, thermoelectrics (TE) can play an important role in recovering energy from waste heat due to its potential in converting heat into electricity, and consequently this effect could be applied in TE power generators or TE refrigerators. The challenge for current state-of-the-art TE devices is the low conversion efficiency. The efficiency of a thermoelectric device is expressed as:

$$
\eta=\frac{T_{H}-T_{C}}{T_{H}}\left[\frac{(1+Z T)^{1 / 2}-1}{(1+Z T)^{1 / 2}+\left(T_{C} / T_{H}\right)}\right]
$$

where $T_{C}$ and $T_{H}$ indicates the temperature of the cold and hot side. The conversion efficiency $\eta$ is determined by the dimensionless thermoelectric figure of merit ZT,

$$
\mathrm{ZT}=\mathrm{S}^{2} \sigma \mathrm{T} /\left(\kappa_{e}+\kappa_{L}\right)
$$

where $\mathrm{S}$ is the Seebeck coefficient, $\sigma$ is the electrical conductivity, $\mathrm{T}$ is the temperature in Kelvin, $\kappa_{\mathrm{e}}$ is the thermal conductivity due to electrons, and $\kappa_{\mathrm{L}}$ is lattice thermal conductivity due to phonons. ${ }^{1}$ Figure 1 shows the simulation result for the dependence of the conversion efficiency $\eta$ on ZT and temperature difference. This graph demonstrates a clear trend: the higher the figure of merit ZT, the higher the conversion efficiency $\eta$ would be. For current state-of-the-art TE devices, the figure of merit ZT value is usually around one, which translates into a conversion efficiency that is usually no higher than $5 \%$. For large-scale practical applications, it is critical to synthesize better TE materials with a figure of merit ZT higher than 2 in order to promote attractive conversion efficiencies for TE devices. Much of the previous work demonstrated that alloying can be used to decrease the lattice thermal conductivity and thus to increase ZT due to mass difference enhanced phonon scattering. ${ }^{2}$ In order to further progress in the development and commercialization of the next generation of thermoelectric devices it is necessary to enhance significantly the figure of merit ZT value. Most recently novel innovative research approaches focused on low dimensional nanostructures, ${ }^{3}$ including quantum wells, ${ }^{4}$ quantum dots, ${ }^{5}$ and superlattices, ${ }^{6}$ in order to improve the figure of merit ZT. Theoretically, the reason why low dimensional nanostructures help to improve ZT was the fact that the quantum confinement effect increases the density of the state near the Fermi level, and consequently

\footnotetext{
*Electrochemical Society Student Member.

**Electrochemical Society Member.

${ }^{\text {z} E-m a i l: ~ h b a u m g a r @ o d u . e d u ~}$
}

increases the thermal power $\left(\mathrm{S}^{2} \sigma\right)$. However experimentally, most of researchers found a reduction of thermal conductivity contributes to the enhancement of ZT. But this was the result from enhanced phonon scattering by numerous interfaces in the lower dimensional structures. ${ }^{7}$

Significant efforts have been made to investigate the IV-VI semiconducting lead chalcogenides, such as PbTe and $\mathrm{PbSe}$, due to their high figure of merit (ZT), good chemical stability, low vapor pressure and high melting point. Therefore $\mathrm{PbTe}$ and $\mathrm{PbSe}$ are promising thermoelectric materials for intermediate temperature applications. PbTe and $\mathrm{PbSe}$ films have been deposited by different techniques, such as pulsed laser deposition (PLD), ${ }^{8}$ metal-organic chemical vapor deposition (MO-CVD),${ }^{9}$ magnetron sputtering, ${ }^{10}$ molecular beam epitaxy $(\mathrm{MBE})^{11}$ and electrical-chemical atomic layer deposition (ALD).12 Thermal ALD is considered to be a novel innovative and competitive

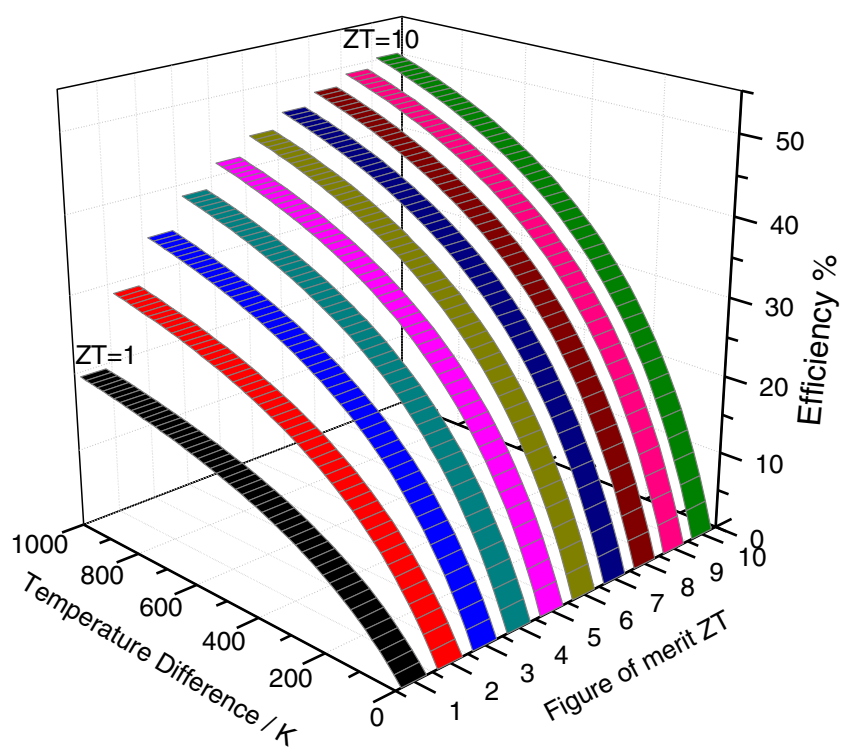

Figure 1. Simulation results of the dependence of TE efficiency on figure of merit (ZT) and Temperature difference. 
method to synthesize $\mathrm{PbTe} / \mathrm{PbSe}$ nanolaminate structures. ${ }^{13}$ Atomic Layer Deposition (ALD) is a surface saturating self-limiting chemical reaction that occurs in each ALD monolayer deposition cycle. ALD technology can precisely control the film layer thickness, stoichiometry, composition, uniformity, and produce sharp interfaces. ALD also can be used to deposit conformal films onto very complex 3-D structures and on the inside of cavities and negative slope surfaces. It is also possible to generate reproducible and well-defined nanolaminate structures. Furthermore, the deposition temperature of ALD is rather low in comparison with other thin film growth techniques.

Porous silicon template substrates and thin porous Si membranes are a relatively novel and versatile material with tunable properties related to their porosity and porous geometry. These porous templates have lower thermal conductivity than bulk silicon due to phononboundary scattering. The thermal conductivity of porous templates is tunable by modulating the porous configuration (staged or aligned), ${ }^{14}$ the geometrical shape of the pores (circular or square) ${ }^{15}$ and the periodicity. ${ }^{16}$ It has been proven by theoretical and experimental work that phonon-boundary scattering in porous materials contributes significantly in reducing phonon thermal conductivity up to two orders of magnitude compared to bulk materials. ${ }^{15}$ When depositing TE film on the porous structure templates, the thermal conductivity of the TE film could be reduced and in term ZT could be enhanced by adjusting the size and periodicity of the porosity pattern and the thickness of the thermoelectric film in relation to the mean free path (MFP) of the phonons of the TE material.

In this study, we present ALD synthesis of $\mathrm{PbTe} / \mathrm{PbSe}$ nanolaminate structures on regular planar bulk silicon wafers and benchmark this conventional configuration against the identical $\mathrm{PbTe} / \mathrm{PbSe}$ nanolaminate structures synthesized inside the periodic lithographically defined pores of porous silicon templates. Physical analysis and surface characterization of the samples has been performed with FE-SEM, XRD, and AFM, while the electrical characterization was performed with Seebeck coefficient measurements of the $\mathrm{ALD} \mathrm{PbTe} / \mathrm{PbSe}$ nanolaminate structures in horizontal and vertical directions.

\section{Experimental}

In this work, multiple layered $\mathrm{PbTe} / \mathrm{PbSe}$ nanolaminates were fabricated by alternately depositing $\mathrm{PbTe}$ and $\mathrm{PbSe}$ layers first on regular planar bulk silicon wafers, and second for direct comparison and benchmarking also inside the myriad pores of porous silicon templates using thermal ALD technology. Lead bis(2,2,6,6tetramethyl-3,5-heptanedionato) $\left(\mathrm{Pb}\left(\mathrm{C}_{11} \mathrm{H}_{19} \mathrm{O}_{2}\right)_{2}\right)$, (trimethylsilyl) telluride $\left(\left(\mathrm{Me}_{3} \mathrm{Si}\right)_{2} \mathrm{Te}\right)$ and (trimethylsilyl) selenide $\left(\left(\mathrm{Me}_{3} \mathrm{Si}\right)_{2} \mathrm{Se}\right)$ were employed as the chemical ALD precursors for lead, tellurium and selenium, respectively. $20 \mathrm{sccm}$ inert $\mathrm{N}_{2}$ was used as a carrier gas to transport the chemical precursors into the ALD reaction chamber. The growth temperature of $\mathrm{PbTe}$ and $\mathrm{PbSe}$ was $150^{\circ} \mathrm{C}$. The solid lead precursor was volatilized at a temperature of $170^{\circ} \mathrm{C}$ with $0.5 \mathrm{~s}$ pulse time, the liquid Te precursor required heating to $42^{\circ} \mathrm{C}$ with $0.03 \mathrm{~s}$ pulse time, and the liquid Se precursor was kept at room temperature with $0.03 \mathrm{~s}$ pulse time. The chamber base pressure was kept at 30 mTorr. The substrates were pretreated with $10 \mathrm{wt} \% \mathrm{HF}$ for $1 \mathrm{~min}$ to remove the native oxide layer. After rinsing in flowing DI water for $5 \mathrm{~min}$, the substrates were then dipped into $\mathrm{H}_{2} \mathrm{O}_{2} / \mathrm{H}_{2} \mathrm{SO}_{4}$ (volume ratio: 1:4) mixed solution for $5 \mathrm{~min}$ to achieve a saturated hydroxyl $\mathrm{OH}^{-}$terminated surface on the substrate, which provided a saturated nucleation site in order to promote the surface chemisorption of the telluride and selenide thin films. The substrates were then rinsed in flowing DI water for 5 min and blow-dried with nitrogen gas. All the chemicals were obtained from Sigma Aldrich and were used without further purification.

In this study, $\mathrm{PbTe} / \mathrm{PbSe}$ nanolaminates films of $10 \mathrm{~nm}$ thickness for each PbTe and PbSe layer were synthesized by ALD and subsequently characterized by X-ray diffraction (XRD) for crystal structure determination. The surface morphology of the ALD TE films were analyzed by field emission scanning electron microscopy (FE-SEM) and

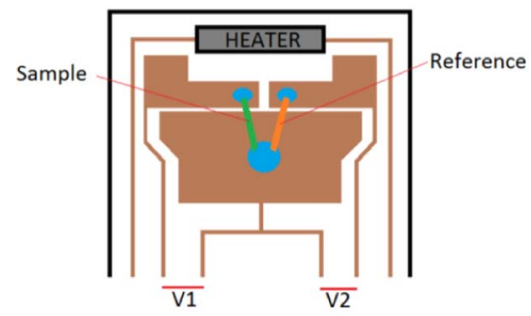

(a)

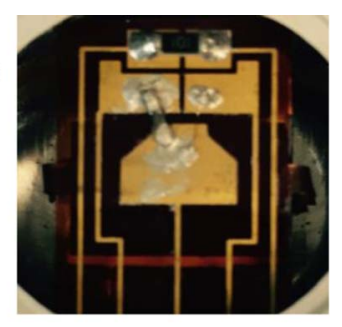

(b)

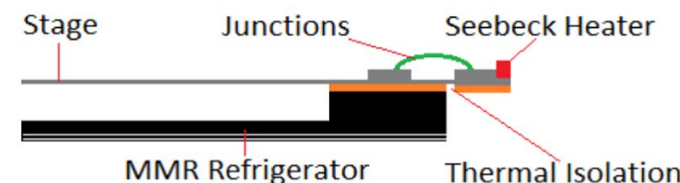

(c)

Figure 2. (a) Top view of Seebeck stage in MMR Seebeck measurement system; (b) Real image of symmetrically mounted our sample and reference sample on the stage; (c) Cross-sectional image of Seebeck stage attached with MMR refrigerator. Figures (a) and (c) reprinted with permission of WWW.MMR-TECH.com.

atomic force microscopy (AFM). In addition for electrical characterization, the Seebeck coefficients of the ALD nanolaminate TE films were measured in the horizontal direction using an MMR Seebeck measurement system.

The ALD TE nanolaminate sample and a reference sample (constantan) were mounted symmetrically on a Seebeck stage in the MMR Seeback system, and were then contacted with leads, as shown in the right-top image in Figure $2 \mathrm{~b}$. The heater insert on top of the stage was computer controlled to heat up the temperature of the hot side, while the cold side was attached to the MMR solid-state refrigerator. These two sides were thermally isolated as shown in bottom image in Figure 2b. There are two pairs of thermocouples on the MMR Seebeck stage, one consists of copper with the sample under test, and the other one is copper with constantan. The Seebeck coefficient was measured differentially by applying a small temperature gradient between the heater and the MMR refrigerator. The voltage response of a particular temperature gradient of the sample under test and of the reference sample were recorded as $V_{1}$ and $V_{2}$. Since the temperature differences between the two sides of the sample and the reference sample were same, the Seebeck coefficient of the sample can be calculated as:

$$
S_{1}=S_{\text {ref }}\left[V_{1}(P) / V_{2}(P)\right]
$$

where $P$ is heating power, $S_{1}$ and $S_{\text {ref }}$ are Seebeck coefficient of the sample under test and the reference sample. To eliminate equipment error and voltage offset, the calculation of the Seebeck coefficient of the sample is modified by collecting the voltage response at two different heater power settings $\left(\mathrm{P}_{1}\right.$ and $\left.\mathrm{P}_{2}\right)$, using the following equation:

$$
\mathrm{S}=S_{\mathrm{Ref}}\left[\frac{V_{1}\left(P_{1}\right)-V_{1}\left(P_{2}\right)}{V_{2}\left(P_{1}\right)-V_{2}\left(P_{2}\right)}\right]
$$

For the Seebeck measurements, the ALD thermoelectric sample was cleaved into a small sized rectangle with a $1 \mathrm{~mm}$ width and a 5 $\mathrm{mm}$ length. Since lead chalcogenide is easily oxidized in air at room temperature, the surface lead oxide layer inhibited Ohmic contacts and instead Schottky diode type exponential I-V behavior between electrodes and the sample was observed. The presence of a surface lead oxide on our PbTe and $\mathrm{PbSe}$ thermoelectric samples therefore affects the Seebeck measurements. In order to achieve good Ohmic contacts, the $\mathrm{PbTe}$ and $\mathrm{PbSe}$ samples were pre-treated by two methods: hydrogen reduction and mechanical scratching of the two sample ends. Following these treatments, Ohmic contacts were achieved by 


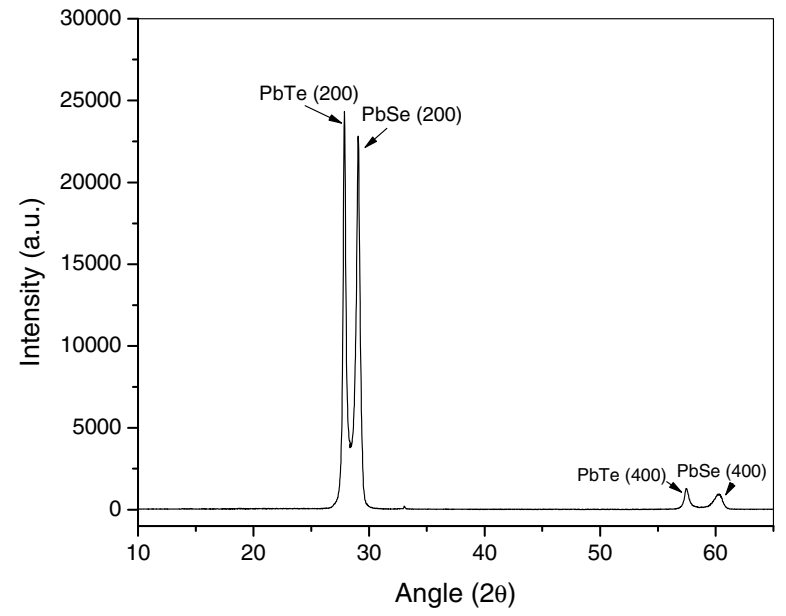

(a)
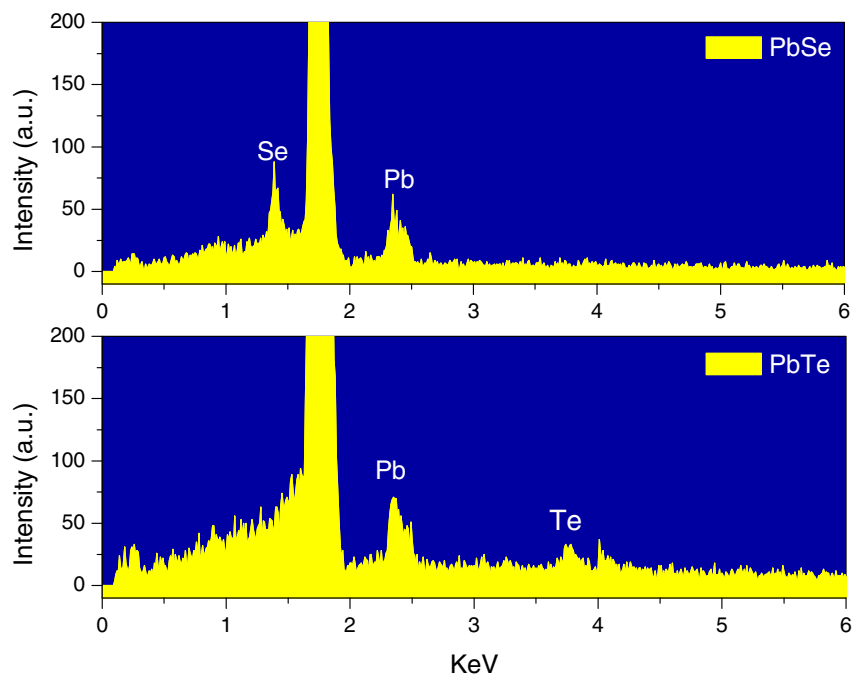

(b)

Figure 3. (a) XRD plot of $\mathrm{PbTe} / \mathrm{PbSe}(10 \mathrm{~nm} / 10 \mathrm{~nm})$ nanolaminates deposited at $150^{\circ} \mathrm{C}$. (b) Energy dispersive X-ray Spectroscopy analysis (EDS) of 1000 cycles of PbSe and 1000 cycles of PbTe samples.

the latter method and by sputter coating Au contact pads on the two ends of the sample. The thickness of the Au contact pads is around 100 $\mathrm{nm}$. Afterwards, the thermoelectric ALD samples with the sputtered electrodes were mounted on the MMR Seebeck stage and contacted with silver paste, as indicated in Figure $2 b$.

\section{Results and Discussion}

The crystal structure of the synthesized ALD films was analyzed by X-ray Diffraction (XRD). Figure 3 displayed the XRD plot of $\mathrm{PbTe} / \mathrm{PbSe}(10 \mathrm{~nm} / 10 \mathrm{~nm})$ nanolaminate structure grown at $150^{\circ} \mathrm{C}$. The Miller indexes marked in the XRD pattern indicate both $\mathrm{PbTe}$ and $\mathrm{PbSe}$ having face centered cubic (FCC) crystal structure. The highest peak in the XRD pattern indicates preferential growth of PbTe and PbSe along the (200) crystal direction, resulting from the lowest sur-

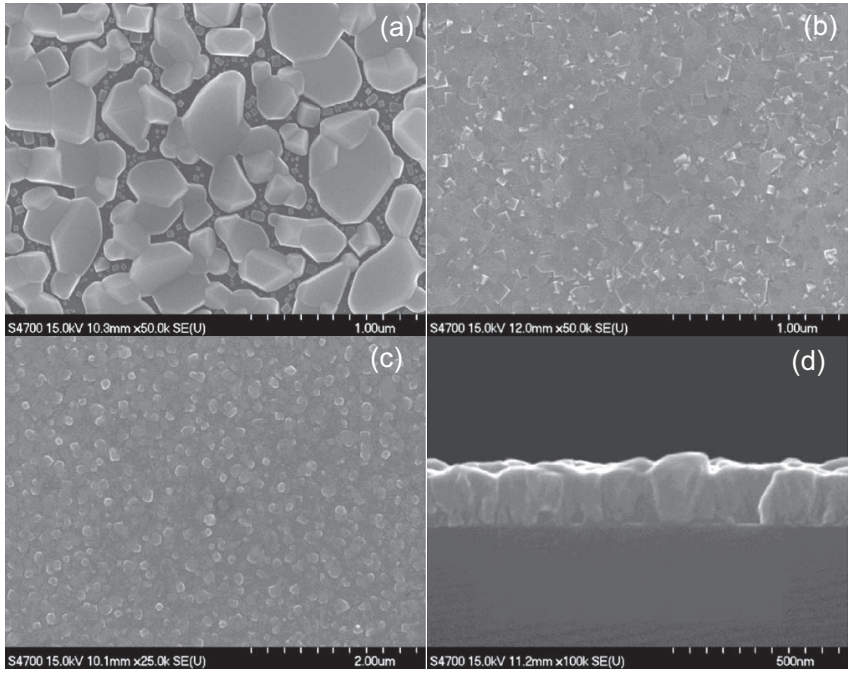

Figure 4. FE-SEM images of (a) 1000 cycles of PbTe film, (b) 1000 cycles of $\mathrm{PbSe}$ film, (c) PbTe/PbSe (10/10 nm) nanolaminates, (d) Cross-section image of $\mathrm{PbTe} / \mathrm{PbSe}(10 / 10 \mathrm{~nm})$ nanolaminates.

face energy in comparison with other orientations. The lattice constant of $\mathrm{PbTe}$ and $\mathrm{PbSe}$ can be calculated by two formulas based on Bragg's law: $\lambda=2 d \sin \Theta$, and $a^{2}=\left(h^{2}+k^{2}+l^{2}\right) d^{2}$. The three letters $\mathrm{h}$, $\mathrm{k}$, and 1 are Miller indexes, and $\mathrm{a}, \mathrm{d}, \lambda$ and $\theta$ are lattice constant, interspace between two adjacent planes, and X-ray wavelength and incident angle of the X-ray, respectively. For (200) crystallographic direction, the lattice constants of PbTe and PbSe are $6.397 \AA$ and $6.159 \AA$, which are compatible with reported values $6.438 \AA$ and $6.128 \AA$, respectively. The lattice mismatch between $\mathrm{PbTe}$ and $\mathrm{PbSe}$ corresponds to $\Delta a / a=3.8 \%$, exhibiting a slight lattice mismatch. The composition and stoichiometry of the sample were observed by energy dispersive X-ray spectroscopy analysis (EDS), shown as Figure $3 \mathrm{~b}$. The result from EDS reveals the chemical element composition in $\mathrm{PbTe}$ and $\mathrm{PbSe}$ films grown at $150^{\circ} \mathrm{C}$ with $1000 \mathrm{ALD}$ deposition cycles. Table I lists the element compositions in $\mathrm{PbTe}$ and $\mathrm{PbSe}$ samples. Both $\mathrm{PbTe}$ and $\mathrm{PbSe}$ are non-stoichiometric being $\mathrm{Pb}$ rich, which indicates the PbTe and $\mathrm{PbSe}$ films are n-type semiconductors, and electrons are the charge carriers. ${ }^{6}$ This was further corroborated by subsequent Seebeck coefficient measurements. The strongest peak at $1.8 \mathrm{keV}$ identifies the Si substrate.

Our previous research reported the ALD growth of $\mathrm{PbTe}$ and $\mathrm{PbSe}$ follows Volmer-Weber island growth mode. ${ }^{17}$ Figure 4a displays the surface morphology of PbTe films on silicon wafer without pre-treatment grown with 1000 ALD deposition cycles. The nucleated $\mathrm{PbTe}$ islands are isolated and exhibit random size and orientation. To achieve complete coverage, we pre-treated substrates as described in the Experimental section in order to add hydroxyl termination $\left(\mathrm{OH}^{-}\right)$ on the surface of the silicon substrate to enhance chemisorption of the Te and Se chemical precursors. Complete and conformal surface coverage with $\mathrm{PbSe}$ films of pre-treated silicon wafers were achieved starting at a thickness of 1000 ALD deposition cycles and above, which is displayed in Figure 4b. The initially isolated nucleated $\mathrm{PbSe}$ islands finally coalesce and combine tightly into a continuous TE film. Figure $4 \mathrm{c}$ shows the surface morphology of a nanolaminate composite structure consisting of 9 bi-layers of $\mathrm{PbTe} / \mathrm{PbSe}$ (each of 10/10 nm) synthesized at $150^{\circ} \mathrm{C}$ on pre-treated planar bulk silicon substrates. The

Table I. The composition of lead, tellurium and selenide in PbTe and PbSe films.

\begin{tabular}{|c|c|c|c|c|}
\hline Samples & $\mathrm{Pb}$ (Weight \%) & Te (Se) (Weight \%) & $\mathrm{Pb}($ Atom \%) & $\mathrm{Te}(\mathrm{Se})($ Atom \%) \\
\hline $\mathrm{TePb}\left(1000\right.$ cys $\left.-170^{\circ} \mathrm{C}\right)$ & $6.53 \pm 1.61$ & $1.98 \pm 0.22$ & $0.86 \pm 0.21$ & $0.42 \pm 0.05$ \\
\hline $\mathrm{SePb}\left(1000\right.$ cys $\left.-170^{\circ} \mathrm{C}\right)$ & $12.07 \pm 0.81$ & $3.68 \pm 0.26$ & $1.88 \pm 0.13$ & $1.5 \pm 0.1$ \\
\hline
\end{tabular}




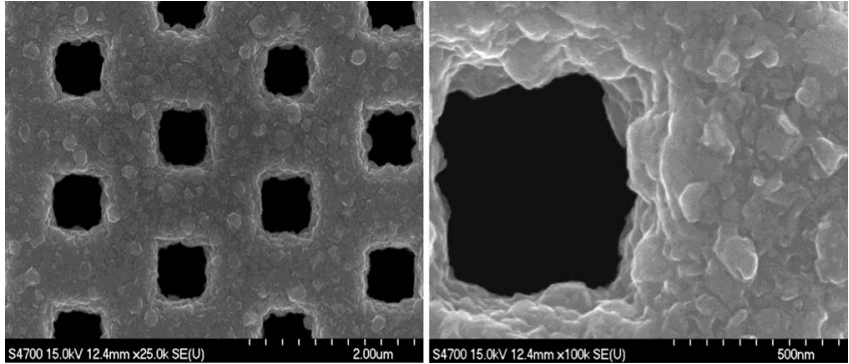

Figure 5. FE-SEM images of 9 bi-layers of $\mathrm{PbTe} / \mathrm{PbSe}(10 / 10 \mathrm{~nm})$ nanolaminates deposited at $150^{\circ} \mathrm{C}$ on microporous silicon template.

FE-SEM micrographs exhibit complete surface coverage of the planar bulk silicon wafer with granular $\mathrm{PbTe} / \mathrm{PbSe}$ nanolaminate layers. The ALD growth of the PbTe and PbSe films still follows the VolmerWeber island growth mode, but with increasing film thickness the isolated nucleated islands coalesce together, which can be seen clearly in the cross-sectional image of the $\mathrm{PbTe} / \mathrm{PbSe}$ nanolaminate structure in Figure 4d. It may be contributed to the saturated nucleation site on the surface of substrate. The thickness of the entire nanolaminate structure is about $200 \mathrm{~nm}$. The FE-SEM micrographs of the nanolaminate structure consisting of 9 bi-layers $\mathrm{PbTe} / \mathrm{PbSe}(10 \mathrm{~nm} / 10 \mathrm{~nm})$ grown on microporous $\mathrm{Si}$ membranes at $150^{\circ} \mathrm{C}$ are illustrated in Figure 5 . The porous silicon template is characterized by an almost square pore size of $2 \mu \mathrm{m}$, with pore walls of $100 \mathrm{~nm}$, and pore depth of $\sim 100 \mu \mathrm{m}$. The SEM micrograph of Figure 5 demonstrates complete coverage of the pore walls with $\mathrm{ALD} \mathrm{PbTe} / \mathrm{PbSe}$ nanolaminate films. In this micrograph the top surface of the pore sidewalls are imaged. This further highlights the significant advantage of ALD technology for coatings of complex 3-D surface morphologies and high aspect ratio pores in porous templates. Figure 6 illustrates a three-dimensional AFM image of the $\mathrm{PbTe} / \mathrm{PbSe}(10 \mathrm{~nm} / 10 \mathrm{~nm})$ nanolaminate structure grown at $150^{\circ} \mathrm{C}$. The surface roughness is $11.37 \mathrm{~nm}$, which indicates that a relatively smooth surface was achieved.

For the Seebeck coefficient measurements, a good Ohmic contact between the electrodes and the semiconducting thermoelectric materials is critical and necessary to obtain correct measurements. It is a known fact that lead chalcogenides are easily oxidized in the air at room temperature. ${ }^{18}$ Therefore, there will be a layer of lead oxide on the surface of the top layer of our $\mathrm{PbTe} / \mathrm{PbSe}$ nanolaminate structure leading to Schottky type diode I-V behavior. In order to achieve good Ohmic contacts between the electrodes and the $\mathrm{PbTe} / \mathrm{PbSe}$ samples, several methods were tried, including hydrogen gas reduction and mechanical scratching to pierce the oxide layer with a needle tip. In order to attempt the reduction of lead oxide, the sample was placed in a furnace quartz tube with flowing forming gas, which is a safe mixture consisting of $95 \%$ nitrogen and $5 \%$ hydrogen gas. The temperature in the furnace tube was heated up to $700^{\circ} \mathrm{C}$ to facilitate the reduction reaction of lead oxide. The reduction temperature was selected based on reported work in the literature. ${ }^{19}$ Au contact pads of about $100 \mathrm{~nm}$ thickness were sputter coated at the two ends of the TE sample immediately after the hydrogen reduction reaction. The mechanical scratching method simply attempted to pierce through the lead oxide in order to expose the PbSe surface locally for contacting purposes. The two ends of the $\mathrm{PbTe} / \mathrm{PbSe}$ sample were scratched by a metal needle tip with several scratching tracks, and then Au contact pads were immediately sputter coated on the two scratched ends. The depth of the scratching is about the thickness of the ALD nanolaminate film $(200 \mathrm{~nm})$. The primary purpose of the scratch method is to break through the surface oxide of the ALD PbTe/PbSe nanolaminate sample, and consequently allow the sputtered Au contact inside the freshly scratched out groove to contact with the nanolaminate material directly. The I-V characteristic curve of three different samples were measured and the results were displayed in Figure $7 \mathrm{a}$. The black line indicates the I-V curve of the sample where the Au coating was sputtered directly on the oxidized sample, without any treatment. This

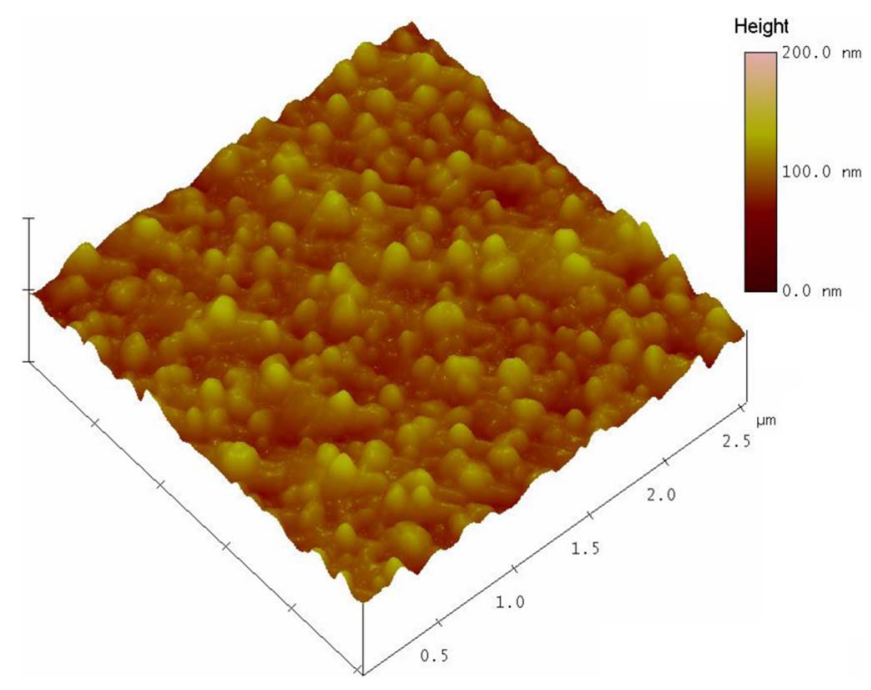

Figure 6. AFM images 9 bi-layers of $\mathrm{PbTe} / \mathrm{PbSe}(10 / 10 \mathrm{~nm})$ nanolaminates deposited at $150^{\circ} \mathrm{C}$ on planar silicon wafer.

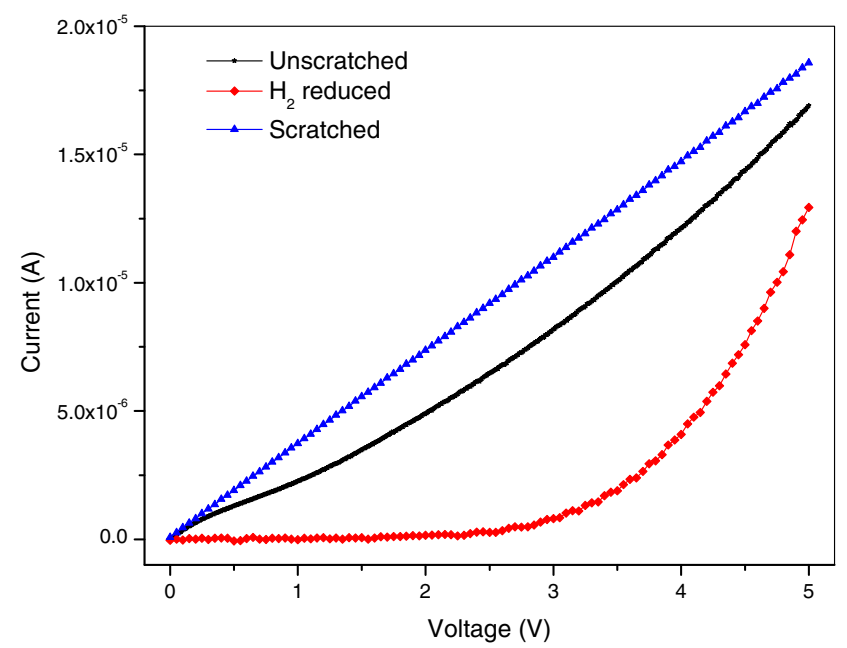

(a)

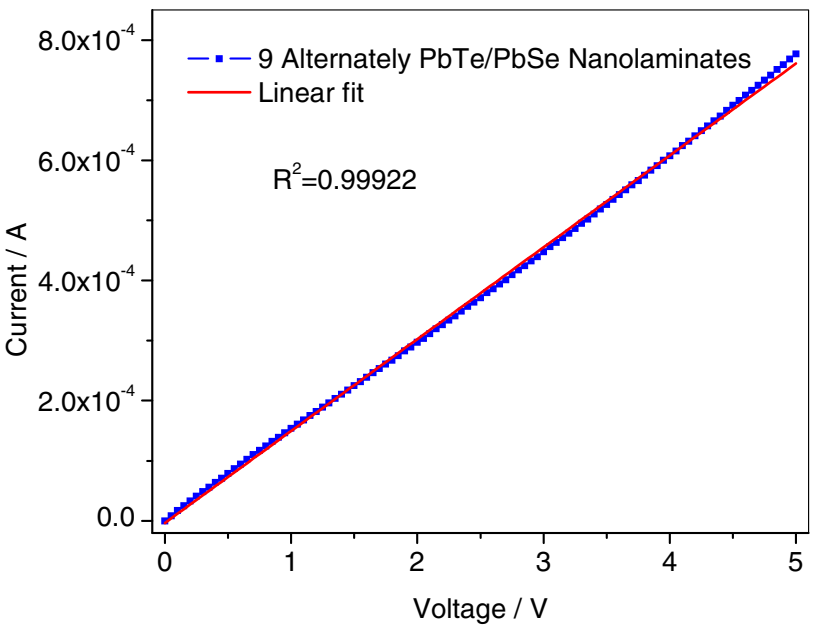

(b)

Figure 7. (a) IV characteristics of triple layered $\mathrm{PbTe} / \mathrm{PbSe} / \mathrm{PbTe}$ (5000/4000/6000 ALD cycles) samples with Au contact on two ends after no treatment (black line with Solid Square), hydrogen reduction (red line with solid rhombus), and scratching (blue line with solid triangle). (b) IV characteristics of 9 bi-layers of $\mathrm{PbTe} / \mathrm{PbSe}(10 / 10 \mathrm{~nm})$ nanolaminates. 
I-V curve indicates Schottky type diode behavior between the Au electrode and the sample due to the presence of an insulating oxide layer. The I-V curve for the sample with Au contact after hydrogen reduction is shown as a red line in Figure 7a. After hydrogen reduction reaction at $700^{\circ} \mathrm{C}$ in forming gas, the sample looks brighter than before, but the low current and the pronounced exponential I-V plot reveals Schottky contact between the Au electrodes and the sample. The forming gas reduction experiment produced a more exponential diode type I-V plot than the original one without any pre-treatment. This may have resulted from oxidation of the sample due to moisture or impurity in the forming gas. Alternatively, the heating temperature of $700^{\circ} \mathrm{C}$ may not be sufficient to enable a hydrogen reduction reaction of $\mathrm{PbTe} / \mathrm{PbSe}$ in our case. Finally, the blue line in Figure 7a shows the $\mathrm{I}-\mathrm{V}$ characteristic of the sample with Au contact electrode following the mechanical scratching method to pierce through the lead oxide. In this case, the linear I-V curve indicates that a very good Ohmic contact was finally achieved between the Au electrodes and the $\mathrm{PbTe} / \mathrm{PbSe}$ film. Therefore, we conclude that mechanical scratching of the surface lead oxide provides a simple, safe and most effective way to get good Ohmic contacts for our $\mathrm{PbTe}$ and $\mathrm{PbSe}$ samples. Figure $7 \mathrm{~b}$ displays the $\mathrm{I}-\mathrm{V}$ curve of $\mathrm{PbTe} / \mathrm{PbSe}$ nanolaminate structure with 9 alternating-layers of 1000 ALD deposition cycles of PbTe and 1000 ALD deposition cycles of $\mathrm{PbSe}$. Both samples were prepared using the oxide scratching method. The ideal linear I-V curve proves that good Ohmic contact between the Au electrodes and the $\mathrm{PbTe} / \mathrm{PbSe}$ TE film was achieved.

To illustrate the effect of the micro-porous silicon template, we measured the temperature dependence of the Seebeck coefficient for $\mathrm{PbTe} / \mathrm{PbSe}$ nanolaminates grown by ALD on both bulk silicon wafers and micro-porous silicon templates. The measurements were conducted over a temperature range from $300 \mathrm{~K}$ to $500 \mathrm{~K}$, and then back-traced from $500 \mathrm{~K}$ to $300 \mathrm{~K}$. The same measurement sequence was repeated again overnight. Figure 8 a displays the dependence of the Seebeck coefficient on temperature for $\mathrm{PbTe} / \mathrm{PbSe}$ nanolaminates grown on bulk silicon wafers. The maximum value of the Seebeck coefficient of the bulk sample with $143.016 \pm 5.500 \mu \mathrm{V} / \mathrm{K}$ was found at $300 \mathrm{~K}$. As the temperature increases from $300 \mathrm{~K}$ to $500 \mathrm{~K}$, the Seebeck coefficient decreases almost linearly. The negative value of the Seebeck coefficient indicates electrons are the majority carriers in the $\mathrm{PbTe} / \mathrm{PbSe}$ nanolaminates. The measured Seebeck coefficient of the thermoelectric nanolaminates deposited on micro-porous silicon templates is shown in Figure 8b. The Seebeck coefficient of the sample synthesized on a micro-porous silicon template exhibits the same temperature dependence compared to the sample deposited on a regular bulk silicon substrate. The Seebeck coefficient decreases consistently from $300 \mathrm{~K}$ to $500 \mathrm{~K}$. The maximum value of the Seebeck coefficient is $370.556 \pm 37.750 \mu \mathrm{V} / \mathrm{K}$ at $300 \mathrm{~K}$. It is noteworthy that the in-plane Seebeck coefficient for our $\mathrm{PbTe} / \mathrm{PbSe}$ nanolaminate structure measured in porous $\mathrm{Si}$ membranes is about 2.5 times larger compared to the value obtained for planar bulk Si substrates. The enhanced Seebeck value is attributed to the presence of the regular array of etched out pores, which provides an additional periodic structuring of the nanolaminate composite sample, while the high porosity of the porous Si template simultaneously reduces the thermal conductivity. This result appears to indicate that the nano-periodic structuring of the surface of thermoelectric samples with porous templates provides an additional parameter that helps to increase the Seebeck Coefficient and ultimately the figure of merit ZT. The dependence of the Seebeck coefficient on temperature exhibits good agreement for both up-trace and down-trace measurements over the temperature range as well as high reproducibility. A negative value of the measured Seebeck coefficient is indicative of n-type material for the $\mathrm{PbTe} / \mathrm{PbSe}$ nanolaminates.

The Seebeck coefficient of all the thermoelectric ALD samples under test reaches a maximum value around room temperature. Our measured temperature dependence of the Seebeck coefficient is different from the one ${ }^{20}$ in which the maximum Seebeck coefficient occurs at $560 \mathrm{~K}$. One of reasons for the discrepancy may be attributed to different thermoelectric film properties due to different deposition

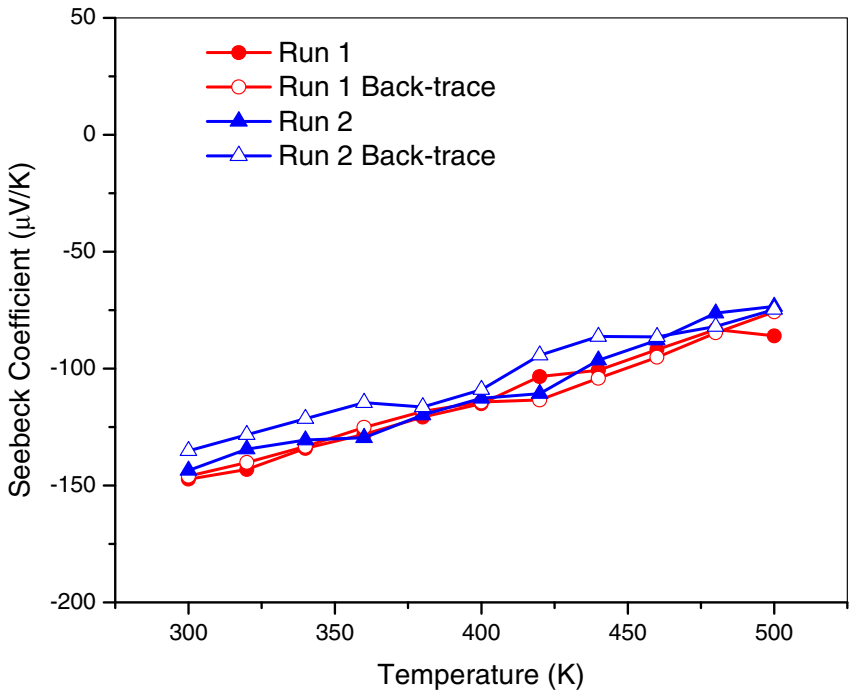

(a)

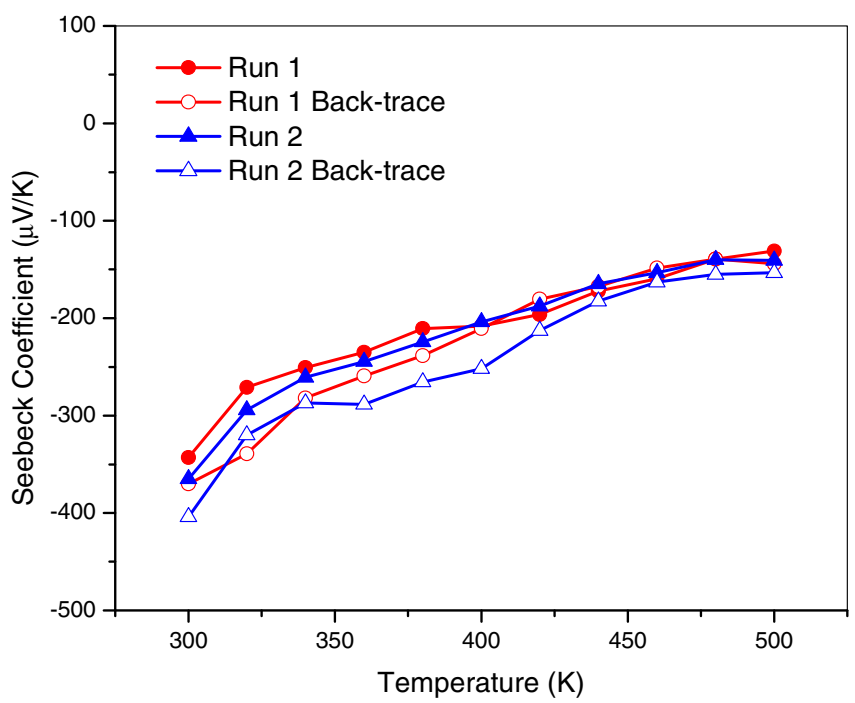

(b)

Figure 8. Seebeck coefficient of 9 bi-layers of $\mathrm{PbTe} / \mathrm{PbSe}(10 / 10 \mathrm{~nm})$ nanolaminates deposited on (a) plain Si substrate and (b) microporous silicon substrate measured by MMR Seebeck measurement system. The measurements were conducted in the temperature range of $300 \sim 500 \mathrm{~K}$.

techniques. It is believed that the maximum Seebeck coefficient occurs when intrinsic conduction begins. Our undoped $\mathrm{PbTe}$ and $\mathrm{PbSe}$ films evidently have lower carrier concentration than doped films, which consequently results in the onset of intrinsic conduction at a lower temperature. ${ }^{21}$

For the Seebeck coefficient measurements in the direction vertical to the surface, our collaborator in MicroXact Inc. reported similar results that the $\mathrm{PbTe} / \mathrm{PbSe}$ nanolaminate structure grown on micro-porous silicon membranes exhibits higher Seebeck coefficients $(78670 \pm 15540 \mu \mathrm{V} / \mathrm{K})$ compared with the thermoelectric nanolaminate structure grown on a planar bulk silicon wafer $(250 \pm 27 \mu \mathrm{V} / \mathrm{K}){ }^{22}$ This porous $\mathrm{Si}$ membrane is fabricated by removing the Si from the back side with $\mathrm{KOH}$ etching until the pores are reached from the back side. From the results of our Seebeck measurements in both the horizontal and vertical directions, the low dimensional ALD nanolaminate structures synthesized inside the pores of porous silicon membranes exhibit higher Seebeck coefficients than previously reported values. This indicates that the added periodic surface structuring resulting from the photolithographically defined regular pore pattern of the 
porous Si membrane aids in further improving the Seebeck coefficient of TE materials. This is attributed to the lower thermal conductivity $\kappa$ in porous $\mathrm{Si}$ membrane structures. The porous silicon membrane we used exhibits a staged square porous configuration, which theoretically yields the lowest thermal conductivity compared to other porous configuration arrangements. ${ }^{15}$ Since the temperature gradient is the driving force for charge carriers moving from hot side to cold side, it can be more lasting in a material with lower thermal conductivity, so that more charged carriers accumulate at cold side leading to larger voltage difference between two sides, and consequently results in a larger Seebeck coefficient.

\section{Conclusions}

This work has demonstrated successful ALD synthesis of low dimensional thermoelectric $\mathrm{PbTe} / \mathrm{PbSe}$ nanolaminate composite materials on native $\mathrm{SiO}_{2}$ covered $\mathrm{Si}$ wafers and microporous silicon membranes by ALD technology. The surface morphology observations indicate heterogeneous nucleation growth of $\mathrm{PbTe} / \mathrm{PbSe}$ nanolaminates during the thermal ALD deposition process rather than classic layer-by-layer growth. The ALD PbTe/PbSe nanolaminates exhibited complete surface coverage on planar bulk silicon wafers and inside the pores of microporous silicon membranes. For the case of porous Si templates, the Seebeck measurements in both the horizontal and vertical directions revealed significant enhancement in Seebeck coefficients for $\mathrm{PbTe} / \mathrm{PbSe}$ nanolaminates synthesized inside strictly periodic lithographically defined porous silicon membranes with values of around $370.556 \pm 37.750 \mu \mathrm{V} / \mathrm{K}$ in horizontal direction and $78670 \pm 15540 \mu \mathrm{V} / \mathrm{K}$ in vertical direction at room temperature. The Seebeck coefficient values seen for the case of planar bulk Si substrates is attributed to the presence of the high density of grain boundaries in our polycrystalline ALD PbTe and PbSe films and the numerous interfaces between the nanolaminate layers that enhance phonon scattering, and hence resulted in a reduction of thermal conductivity. However, a much larger enhanced Seebeck coefficient was measured for the case of ALD nanolaminate coatings of porous $\mathrm{Si}$ templates and thin porous membranes, which concomitantly reduce the thermal conductivity even more due to the high porosity with less Si remaining. This approach of synthesizing layered nanolaminates of polycrystalline $\mathrm{PbTe}$ and $\mathrm{PbSe}$ thermoelectric material by atomic layer deposition (ALD) technology promises a high potential for further improvements of the Seebeck coefficient and the figure of merit ZT.

\section{Acknowledgments}

This work was partially funded by the US Air Force Office of Scientific Research (AFOSR) under subcontract No. 01_AFOSRTE2 and Prime Contract FA9550-12-C-0076; project title: "Next Generation Thermoelectric Devices II".

\section{References}

1. D. M. Rowe, CRC handbook of thermoelectrics., CRC press (1995).

2. Z. G. Chen, G. Han, L. Yang, L. Cheng, and J. Zou, Progress in Natural Science: Materials International, 22(6), 535 (2012)

3. C. J. Vineis, A. Shakouri, A. Majumdar, and M. G. Kanatzidis, Advanced Materials, 22(36), 3970 (2010).

4. R. Venkatasubramanian, E. Siivola, T. Colpitts, and B. O'quinn, Nature, 413(6856), 597 (2001).

5. T. C. Harman, P. J. Taylor, D. L. Spears, and M. P. Walsh, Eighteenth International Conference on. 1999. IEEE (1999).

6. T. C. Harman, P. J. Taylor, M. P. Walsh, and B. E. LaForge, Science, 297(5590), 2229 (2002).

7. M. S. Dresselhaus, G. Chen, M. Y. Tang, R. G. Yang, H. Lee, D. Z. Wang, Z. F. Ren, J. P. Fleurial, and P. Gogna, Advanced Materials, 19(8), 1043 (2007).

8. A. Dauscher, M. Dinescu, O. M. Boffoué, A. Jacquot, and B. Lenoir, Thin Solid Films, 497(1), 170 (2006).

9. T. Trindade and P. O. Brien, Chemical Vapor Deposition, 3(2), 75 (1997).

10. A. Jdanov, J. Pelleg, Z. Dashevsky, and R. Shneck, Materials Science and Engineering: $B, \mathbf{1 0 6}(1), 89$ (2004).

11. H. Wu, C. Cao, J. Si, T. Xu, H. Zhang, H. Wu, J. Chen, W. Shen, and N. Dai, Journal of applied physics, 101(10), 103505 (2007).

12. D. Banga, Y. G. Kim, and J. Stickney, Journal of The Electrochemical Society, 158(2), D99 (2011).

13. D. Gu, H. Baumgart, K. Tapily, P. Shrestha, G. Namkoong, X. Ao, and F. Müller, Nano Research, 4(2), 164 (2011).

14. D. Song and G. Chen, Applied physics letters, 84(5), 687 (2004).

15. G. Romano and J. C. Grossman, Applied Physics Letters, 105(3), 033116 (2014).

16. J. Nakagawa, Y. Kage, T. Hori, J. Shiomi, and M. Nomura, Applied Physics Letters, 107(2), 023104 (2015).

17. K. Zhang, A. R. Pillai, K. Bollenbach, D. Nminibapiel, W. Cao, H. Baumgart, T. Scherer, V. S. K. Chakravadhanula, C. Kübel, and V. Kochergin, ECS Journal of Solid State Science and Technology, 3(6), P207 (2014).

18. C. Gautier, M. Cambon-Muller, and M. Averous, Applied surface science, 141(1), 157 (1999).

19. A. Adler, Process of gaseous reducing lead oxide employing an agent to maintain lead in particulate form. Google Patents (1966).

20. C. J. Vineis, T. C. Harman, S. D. Calawa, M. P. Walsh, R. E. Reeder, R. Singh, and A. Shakouri, Physical Review B, 77(23), 235202 (2008).

21. B. G. Streetman and S. Banerjee, Solid state electronic devices, 5, Prentice Hall New Jersey (2000).

22. B. L. Geist, M. Zaynetdinov, K. Myers, K. Zhang, X. Chen, A. R. Pillai, H. Baumgart, H. D. Robinson, and V. Kochergin, MRS Proceedings. Vol. 1735, p. mrsf14-1735, Cambridge Univ Press (2015). 